\title{
Virtual education in neurosurgery during the COVID-19 pandemic
}

\author{
Tyler Lazaro, MD, ${ }^{1}$ Visish M. Srinivasan, MD, ${ }^{1}$ Maryam Rahman, MD, MS, ${ }^{3}$ Ashok Asthagiri, MD, ${ }^{4}$ \\ Garni Barkhoudarian, MD, ${ }^{5}$ Lola B. Chambless, MD, ${ }^{6}$ Peter Kan, MD, ${ }^{7}$ Ganesh Rao, MD, ${ }^{1}$ \\ Brian V. Nahed, MD, MSc, ${ }^{8}$ and Akash J. Patel, MD ${ }^{1,2,9}$
}

Departments of ${ }^{1}$ Neurosurgery and ${ }^{2}$ Otolaryngology-Head and Neck Surgery, Baylor College of Medicine, Houston, Texas; ${ }^{3}$ University of Florida College of Medicine, Gainesville, Florida; ${ }^{4}$ John Wayne Cancer Institute at Providence Saint John's Health Center, Santa Monica, California; ${ }^{5}$ University of Virginia School of Medicine, Charlottesville, Virginia; ${ }^{6}$ Department of Neurosurgery, Vanderbilt University School of Medicine, Nashville, Tennessee; 'Department of Neurosurgery, University of Texas Medical Branch School of Medicine, Houston, Texas; ${ }^{8}$ Department of Neurosurgery, Harvard Medical School, Boston, Massachusetts; and ${ }^{9} \mathrm{Jan}$ and Dan Duncan Neurological Research Institute, Texas Children's Hospital, Houston, Texas

OBJECTIVE Neurosurgical education in the US has changed significantly as a consequence of the novel coronavirus (COVID-19) pandemic. Institutional social distancing requirements have resulted in many neurosurgical programs utilizing video conferencing for educational activities. However, it is unclear how or if these practices should continue after the pandemic. The objective of this study was to characterize virtual education in neurosurgery and understand how it should be utilized after COVID-19.

METHODS A 24-question, 3-part online survey was administered anonymously to all 117 US neurosurgical residency programs from May 15, 2020, to June 15, 2020. Questions pertained to the current use of virtual conferencing, preferences over traditional conferences, and future inclinations. The Likert scale $(1=$ strongly disagree, $3=$ neutral, $5=$ strongly agree) was used. Comparisons were calculated using the Mann-Whitney U-test. Statistical significance was set at 0.05 .

RESULTS One-hundred eight responses were recorded. Overall, 38 respondents (35.2\%) were attendings and 70 $(64.8 \%)$ were trainees. Forty-one respondents (38.0\%) indicated attending 5-6 conferences per week and $70(64.8 \%)$ attend national virtual conferences. When considering different conference types, there was no overall preference (scores <3) for virtual conferences over traditional conferences. In regard to future use, respondents strongly agreed that they would continue the practice at some capacity after the pandemic (median score 5). Overall, respondents agreed that virtual conferences would partially replace traditional conferences (median score 4), whereas they strongly disagreed with the complete replacement of traditional conferences (median score 1). The most common choices for the partial replacement of tradition conferences were case conferences $(59 / 108,55 \%)$ and board preparation $(64 / 108,59 \%)$. Lastly, there was a significant difference in scores for continued use of virtual conferencing in those who attend nationally sponsored conferences (median score 5, $n=70$ ) and those who do not (median score 4, $n=38 ; U=1762.50, z=2.97, r=0.29, p=$ 0.003).

CONCLUSIONS Virtual conferences will likely remain an integral part of neurosurgical education after the COVID-19 pandemic has abated. Across the country, residents and faculty report a preference for continued use of virtual conferencing, especially virtual case conferences and board preparation. Some traditional conferences may even be replaced with virtual conferences, in particular those that are more didactic. Furthermore, nationally sponsored virtual conferences have a positive effect on the preferences for continued use of virtual conferences.

https://thejns.org/doi/abs/10.3171/2020.9.FOCUS20672

KEYWORDS virtual; education; e-medicine; neurosurgery residency 
$\mathrm{T}$ HE novel coronavirus of 2019 (COVID-19) has fundamentally changed the way neurosurgery is practiced across the US. As COVID-19 cases surged in the spring of 2020, elective cases across the country decreased by more than $50 \%$ at many institutions. ${ }^{1}$ Social distancing and restrictions on gathering have affected neurosurgical training. In addition to the impact on trainee case volumes, didactic education has also changed significantly. Since the start of the pandemic, neurosurgical education has been overhauled and many academic neurosurgical programs have turned to virtual learning, with the use of established video conferencing platforms. ${ }^{2}$

The Congress of Neurological Surgeons (CNS), the AANS, and academic institutions have also sought to mitigate the impact of the COVID-19 pandemic on neurosurgical education with widely available virtual conferences. As a result, neurosurgery trainees and faculty have had increased access to new content on a weekly basis. At the primary site of this study (Baylor College of Medicine), this has resulted in greater access to educational materials than prior to the pandemic.

While many neurosurgery programs are practicing virtual conferencing out of necessity during the COVID-19 pandemic, it remains unclear how this newly established mode of education will continue moving forward. We sought to characterize the current practices of virtual education in academic neurosurgery and understand how this type of learning should be continued after the COVID-19 pandemic has abated.

\section{Methods \\ Online Survey}

A 24-question online survey was sent to 117 neurosurgery training programs in the US, as identified by the Electronic Residency Application Service official site for neurosurgery. Surveys were sent electronically via email to the program coordinators for each residency program, who were asked to disseminate the survey link to all postgraduate trainees and faculty in the department. Invitations were sent weekly for 4 consecutive weeks from May 15, 2020, to June 15, 2020.

The survey was composed of three parts. Part I pertained to demographic information, such as training year, age, time zone, current use of virtual conferencing platforms, and which types of conferences they have participated in. Part II gauged participants' preferences of virtual conferences in comparison to traditional conferences based on the Likert item 5-point response scale $(1=$ strongly disagree, $3=$ neutral, $5=$ strongly agree). Conference types included case conferences, department grand rounds, visiting professors, board preparation didactics, morbidity and mortality conferences, and journal club. Part III was designed to determine participant preferences for continued virtual conferences once social distancing requirements and restrictions on public gatherings are eased. Questions were multiple choice regarding the types of traditional conferences that could be replaced by virtual conferences in some capacity. Many of these questions allowed for the selection of multiple responses for each participant (i.e., "check all that apply").
TABLE 1. Survey respondent demographics

\begin{tabular}{|c|c|}
\hline Variable & $\begin{array}{c}\text { No. of } \\
\text { Responses (\%) }\end{array}$ \\
\hline \multicolumn{2}{|l|}{ Training level } \\
\hline Attending & $38(35.2)$ \\
\hline Trainee (fellows and residents) & $70(64.8)$ \\
\hline PGY-1 & $13(18.6)$ \\
\hline PGY-2 & $9(12.9)$ \\
\hline PGY-3 & $11(15.7)$ \\
\hline PGY-4 & $5(7.1)$ \\
\hline PGY-5 & $11(15.7)$ \\
\hline PGY-6 & $7(10.0)$ \\
\hline PGY-7 & $10(14.3)$ \\
\hline Fellow & $4(5.7)$ \\
\hline \multicolumn{2}{|l|}{ Age, yrs } \\
\hline $20-30$ & $2(1.9)$ \\
\hline $31-40$ & $25(23.1)$ \\
\hline $41-50$ & $52(48.1)$ \\
\hline $51-60$ & $11(10.2)$ \\
\hline $61-70$ & $9(8.3)$ \\
\hline$>70$ & $9(8.3)$ \\
\hline \multicolumn{2}{|l|}{ Time zone } \\
\hline Eastern & $46(42.6)$ \\
\hline Central & $54(50.0)$ \\
\hline Mountain & $1(0.9)$ \\
\hline Pacific & $7(6.5)$ \\
\hline \multicolumn{2}{|l|}{ Primary conferencing platform } \\
\hline Microsoft Teams & $1(0.9)$ \\
\hline Webex & $4(3.7)$ \\
\hline Zoom & $103(95.4)$ \\
\hline \multicolumn{2}{|c|}{ No. of conferences attended per week } \\
\hline $1-2$ & $19(17.6)$ \\
\hline $3-4$ & $33(30.6)$ \\
\hline $5-6$ & $41(38.0)$ \\
\hline$>6$ & $15(13.9)$ \\
\hline \multicolumn{2}{|c|}{ Attendance of nationally available conferences } \\
\hline Yes & $70(64.8)$ \\
\hline No & $38(35.2)$ \\
\hline \multicolumn{2}{|c|}{ No. of technical conference issues per session } \\
\hline 0 & $42(38.9)$ \\
\hline $1-2$ & $55(50.9)$ \\
\hline $3-4$ & $6(5.6)$ \\
\hline $5-6$ & $1(0.9)$ \\
\hline$>6$ & $4(3.7)$ \\
\hline
\end{tabular}

PGY = postgraduate year.

\section{Statistical Analysis}

Data analysis was conducted using SPSS Statistics (version 27, IBM Corp.) and Microsoft Excel (Microsoft Corp.) to create graphic representations. The median was used as the measure of central tendency for all ordinal Likert data. Comparisons were made using nonparametric 


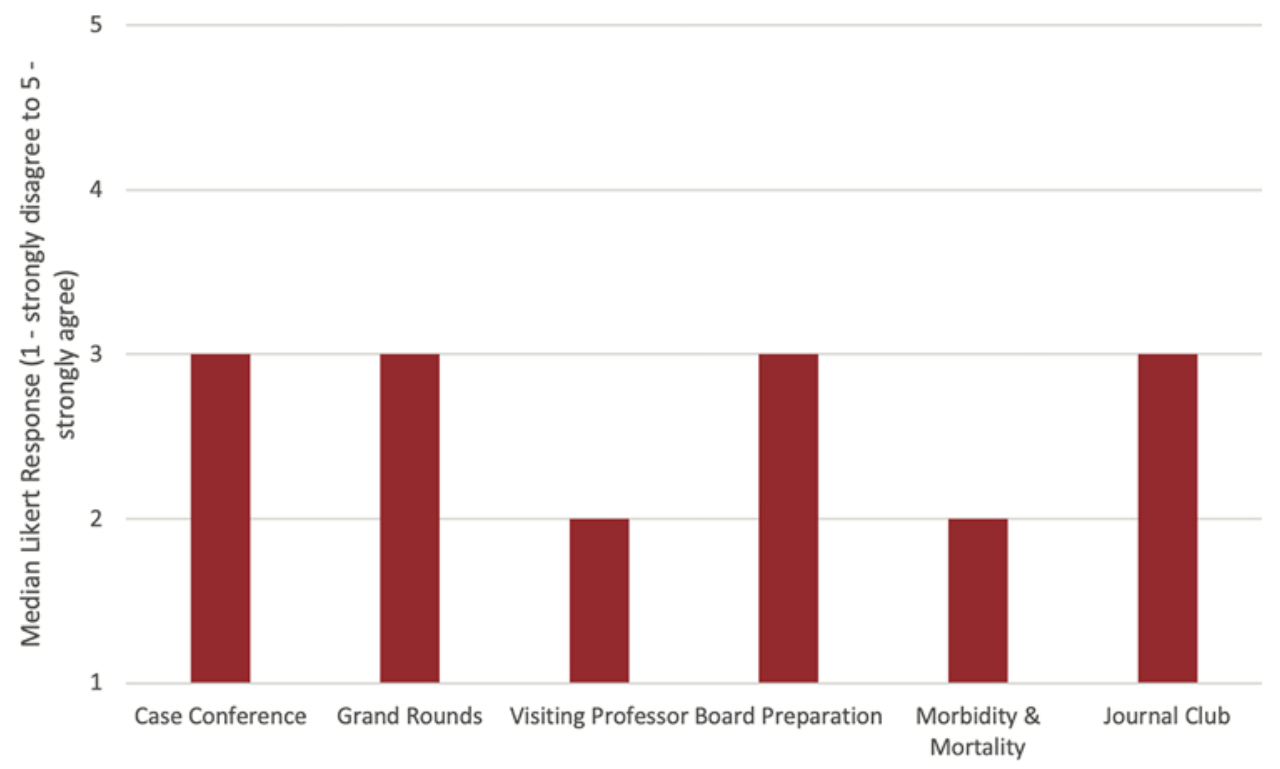

FIG. 1. Bar graph showing preferences of virtual conferences over traditional conferences.

analyses, specifically the Mann-Whitney U-test. A p value $\leq 0.05$ was chosen as the statistically significant threshold to reject the null hypothesis.

\section{Results}

Of 117 programs that were sent the survey, participants from 28 programs (24\%) responded. In total, there were 108 respondents. Of all submitted surveys, 28 contained missing responses for Likert data in part II, and were excluded from the analysis for each independent question.

\section{Part I}

Table 1 summarizes the results from part I of the survey. Most participants were neurosurgery trainees, with $70(64.8 \%)$ being residents or fellows. Attending neurosurgeons accounted for 38 (35.2\%) of the total responses. Fifty-two respondents (48\%) were between the ages of 41 and 50. Respondents were predominantly from central or eastern time zones, with 54 and 46 participants, respectively (50\% and $42.6 \%)$.

The most frequently used virtual communications platform was Zoom (103 participants, 95.4\%), followed by Webex (4 participants, 3.7\%), then Microsoft Teams (1 participant, $0.9 \%)$. Forty-one participants $(38 \%)$ attended 5-6 conferences per week. The majority of participants (n $=70,64.8 \%$ ) also attended nationally available conferences, such as the CNS Virtual Visiting Professor and Weil Cornell Neurosurgery's Virtual Global Spine Conference (listed in the comments for individual surveys). Technical issues during these conferences were not common, with 42 participants $(38.9 \%)$ reporting no technical issues per session and 55 participants (50.9\%) reporting 1-2.

\section{Part II}

The internal reliability of the survey questions was satisfactory with a Cronbach's alpha of 0.748 , after 1 question was excluded from the survey to increase consistency (see question 18 in Supplementary Fig. 1). Overall, when participants were asked which conference types are preferred in a virtual format over a traditional format, case conference, grand rounds, board preparation, and journal club had a median Likert response of 3 (neutral; Fig. 1). Most respondents preferred traditional conferences for visiting professor and morbidity and mortality (Likert score 2, disagree). However, when asked if participants would continue to use virtual conferences given their current experiences after the pandemic has abated, the median participant response was 5 (strongly agree; Fig. 2). Furthermore, when asked if virtual conferences would partially replace traditional conferences after the pandemic had abated, the median response was 4 (agree).

\section{Part III}

With regard to preferences for future use of virtual conferencing, participants preferred early morning (7:00 9:00 Am local time) and evening (after 5:00 PM local time), with 56 and 58 responses, respectively (Fig. 3). Overall, most participants felt that $25 \%-50 \%$ of conferences could be conducted virtually only after the pandemic ends (Fig. 4). Moreover, by conference type, participants felt that case conferences (59 responses), research seminars (58 responses), and board preparation (64 responses) could at least be partially replaced by virtual conferences only (Fig. 5). In contrast, $50 \%$ of participants felt that no conference could be completely replaced by virtual conferences only (54 responses), but some felt that research seminars (28 responses) and board preparation (36 responses) could be completely replaced.

\section{Comparison of Groups in Continued Use of Virtual Conferencing}

Several comparisons were made between participant 


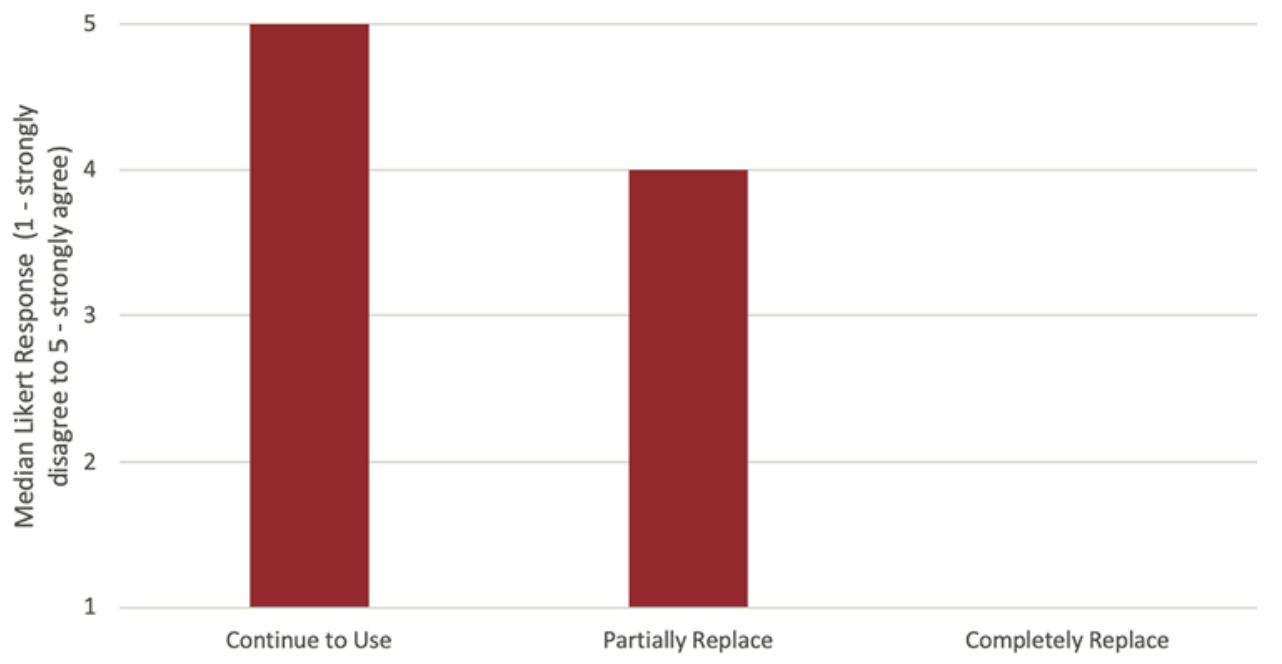

FIG. 2. Bar graph showing preferences of continued use of virtual conferencing.

characteristics and preferences in virtual conferencing. The preferences to continue to use virtual conferencing, as reported by the Likert data from Part II, were analyzed to see if there were any differences between participants of differing training status or those who attend national conferences (Table 2). A Mann-Whitney U-test showed that there were no significant differences in likeliness to continue to use virtual conferencing between trainees (residents and fellows; median $4, \mathrm{n}=70$ ) and attendings (me$\operatorname{dian} 4.5, \mathrm{n}=38 ; \mathrm{U}=1436.00, \mathrm{z}$ score $=0.73, \mathrm{r}=0.07, \mathrm{p}=$ $0.47)$. Examining participants who attend national virtual conferences, however, there was a significant difference in likeliness to continue to use virtual conferencing between attendees (median 5, $\mathrm{n}=70$ ) and nonattendees (median 4, $\mathrm{n}=38 ; \mathrm{U}=1762.50, \mathrm{z}$ score $=2.97, \mathrm{r}=0.29, \mathrm{p}=0.003)$.

\section{Discussion}

This descriptive survey study was designed to characterize the current use of virtual conferencing for neurosurgical education at academic institutions during the COVID-19 pandemic. In addition, we sought to determine preferences to guide the continued use of virtual conferences after the pandemic. Overall, our results suggest that virtual conferencing, while a necessity during the pandemic, will likely continue as a component of neurosurgical education in the future.

Most of the participants of the study were trainees who use web conferencing to attend virtual conferences multiple times per week. The majority also regularly attend nationally sponsored virtual conferences. Although the use of vir-

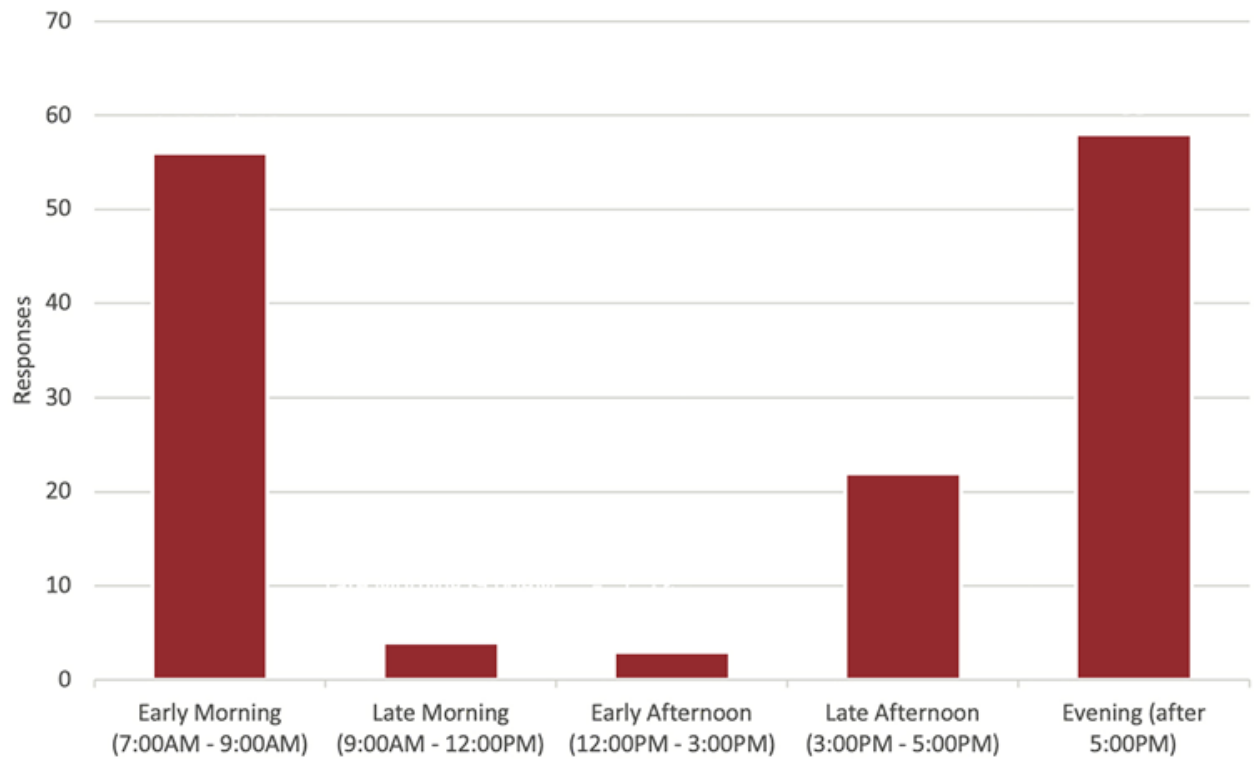

FIG. 3. Graph of preferences of time of day for future virtual conferences. 


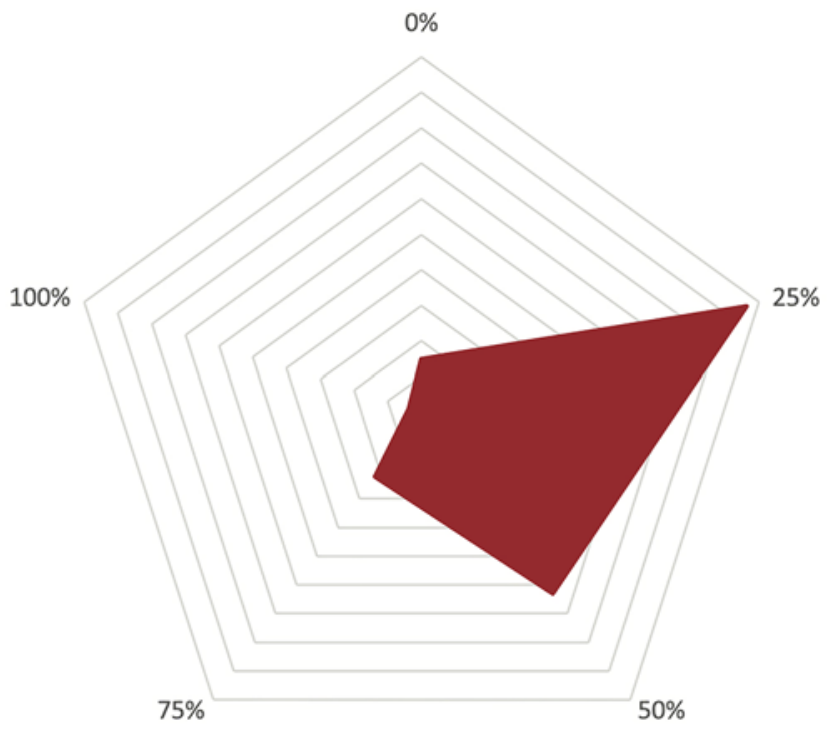

FIG. 4. Radar chart of preferences for proportion of conferences to be virtual only in the future. Shaded areas of the chart closer to the corners indicate a greater number of responses.

tual conferencing technologies is common, this study did not show a definitive preference for virtual conferences over traditional ones across conference categories. However, there was a weak preference for traditional conferences pertaining to visiting professors and morbidity and mortality.

Although the current preferences for virtual conferences over traditional ones are neutral, participant responses regarding the future use of virtual conferencing express a strong preference for continued utilization, and even partial replacement of traditional conferences with virtual ones. Most participants responded with a preference to replace traditional case conferences, research seminars, and board preparation with virtual conferences in some capacity. However, the majority responded unfavorably to replacing any conference completely. These results would suggest that more didactic-type conferences with greater involvement in resident education could more likely be replaced with virtual options.

Regarding the future utilization of virtual platforms for neurosurgical education, participants who regularly attended nationally sponsored virtual conferences were more likely to prefer continued virtual conferencing in the future, with a moderate effect size. ${ }^{3}$ Attendings and trainees did not differ in their preferences to continue virtual conferencing. Overall, these data support continued nationally available virtual conferences, such as webinars and virtual grand grounds through organizations such as the CNS and AANS.

While virtual conferencing technologies have existed for years, they are now being utilized to the fullest extent out of necessity during the COVID-19 pandemic. In fact, all medical fields looking to offer continued medical education are trying to optimize virtual opportunities. In neurosurgery, these data support its continued use and indicate a possible shift in neurosurgical education toward the utilization of virtual platforms after the pandemic, likely as a result of the accessibility, low cost, and convenience of virtual conferencing. In addition, because continued nationally sponsored virtual conferences are clearly well attended based on these data, neurosurgical organizations should continue to offer these virtual opportunities for continued medical education, which could also serve to disseminate critical knowledge updates and research breakthroughs in the future, even after the pandemic.

To the best of our knowledge, this is the first study evaluating virtual education in neurosurgery to this point in the COVID-19 pandemic. Furthermore, we aimed to quantify the preferences for current and continued use of virtual conferences with Likert scale responses. However, the use of Likert data has inherent weaknesses, including question bias, although the internal reliability of the survey data was high. ${ }^{4}$ Additionally, the study suffered from low recruitment. Of 117 programs in the country, partici-

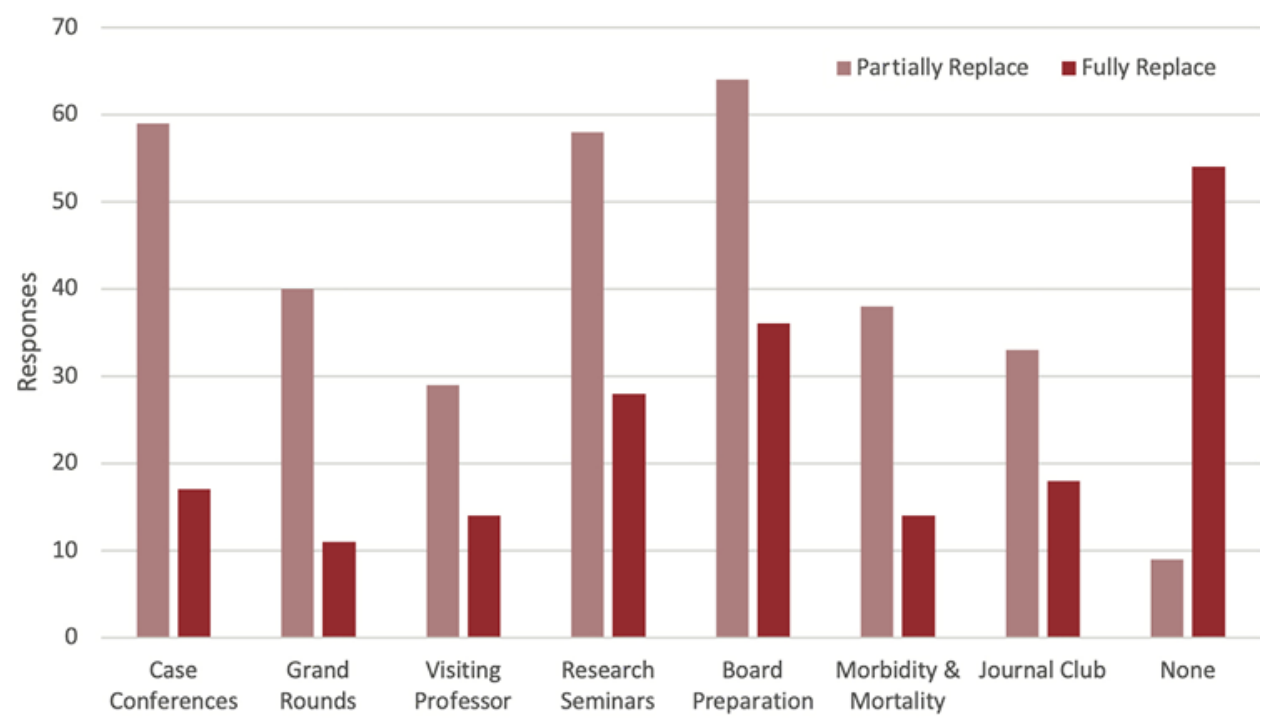

FIG. 5. Bar graph of preferences for partial and full replacement of traditional conferences with virtual conferences. 
TABLE 2. Comparison of preference of continued use of nationally sponsored virtual conferences by training status and attendance

\begin{tabular}{lccccc}
\hline \multicolumn{1}{c}{ Comparison } & Mann Whitney U-test $(U)$ & z Score & No. (Median) & Effect Size (r) & $p$ Value \\
\hline $\begin{array}{l}\text { Continued use of virtual conferencing among train- } \\
\text { ees vs attendings }\end{array}$ & 1436.00 & 0.73 & $\begin{array}{c}\text { Trainees }=70(4), \\
\text { Attendings }=38(4.5)\end{array}$ & 0.07 & 0.47 \\
\hline $\begin{array}{l}\text { Continued use of virtual conferencing among those } \\
\text { who have attended national virtual conferences \& } \\
\text { those who have not }\end{array}$ & 1762.50 & 2.97 & $\begin{array}{c}\text { Attendees }=70(5), \\
\text { Nonattendees }=38(4)\end{array}$ & 0.29 & $0.003^{*}$ \\
\hline
\end{tabular}

* Statistically significant.

pants from only 28 programs completed surveys. Also, most participants were from the central and eastern time zones, and therefore those from the western and mountain time zones were underrepresented.

Future studies are necessary to determine the effectiveness of specific virtual curricula, such as simulation laboratories, given the significantly decreased surgical case volumes since the start of the pandemic. Simulation is well established in general surgical training as a method of developing skills transferable to the operative setting. 5,6 In recent studies, simulation and augmented reality have also been explored in neurosurgical training with promising results. ${ }^{7.8}$ Thus, studies looking into how these virtual education methods can contribute to neurosurgery training should be considered to help enhance the operative experience during the COVID-19 pandemic.

Lastly, further research is needed to assess the impact of virtual education on global neurosurgical training. Trainees from resource-poor countries often struggle to obtain learning materials and basic surgical instruction. ${ }^{9,10}$ As a result, they are forced to obtain neurosurgical training from nontraditional sources, given limited access to content or travel. Therefore, it is likely that these disadvantaged training programs benefited greatly from the collection of virtual lectures made easily accessible during the COVID-19 pandemic. Ultimately, if the data from this study are any indication, continued availability of virtual conferencing beyond the pandemic would help to further a global neurosurgical community and inclusive academic environment.

\section{Conclusions}

Virtual conferences will likely remain an integral part of neurosurgical education after the COVID-19 pandemic has abated. Across the country, residents and faculty appear to have some preference for continued use of virtual conferencing, especially virtual case conferences and board preparation. Some traditional conferences may even be replaced with virtual conferences, particularly those that are more didactic. Furthermore, nationally sponsored virtual conferences have a positive effect on the continued use of virtual conferences.

\section{References}

1. Jean WC, Ironside NT, Sack KD, et al. The impact of COVID-19 on neurosurgeons and the strategy for triaging non-emergent operations: a global neurosurgery study. Acta Neurochir (Wien). 2020;162(6):1229-1240.
2. Bray DP, Stricsek GP, Malcolm J, et al. Letter: Maintaining neurosurgical resident education and safety during the COVID-19 pandemic. Neurosurgery. 2020;87(2):E189-E191.

3. Cohen J. Statistical Power Analysis for the Behavioral Sciences. 2nd ed. Routledge; 1988.

4. Likert R. A technique for the measurement of attitudes. Arch Psychol. 1932;22(140):1-55.

5. Seymour NE, Gallagher AG, Roman SA, et al. Virtual reality training improves operating room performance: results of a randomized, double-blinded study. Ann Surg. 2002;236(4):458-464.

6. Sturm LP, Windsor JA, Cosman PH, et al. A systematic review of skills transfer after surgical simulation training. Ann Surg. 2008;248(2):166-179.

7. Breese R, Piazza M, Quinsey C, Blatt JE. Tactile skill-based neurosurgical simulators are effective and inexpensive. World Neurosurgery. 2020:319-326.

8. Cho J, Rahimpour S, Cutler A, et al. Enhancing reality: a systematic review of augmented reality in neuronavigation and education. World Neurosurg. 2020;139:186-195.

9. Nicolosi F, Rossini Z, Zaed I, et al. Neurosurgical digital teaching in low-middle income countries: beyond the frontiers of traditional education. Neurosurg Focus. 2018;45(4):E17.

10. Campain NJ, Kailavasan M, Chalwe M, et al. An evaluation of the role of simulation training for teaching surgical skills in sub-Saharan Africa. World J Surg. 2018;42(4):923-929.

\section{Disclosures}

Dr. Barkhoudarian reports being a consultant to Vascular Technologies, Inc. Dr. Nahed reports being a cofounder of React Neuro.

\section{Author Contributions}

Conception and design: Lazaro, Srinivasan, Patel. Acquisition of data: Lazaro. Analysis and interpretation of data: Lazaro. Drafting the article: Lazaro. Critically revising the article: all authors. Reviewed submitted version of manuscript: Patel, Lazaro. Statistical analysis: Lazaro. Administrative/technical/material support: Lazaro. Study supervision: Patel.

\section{Supplemental Information}

Videos

Video Abstract. https://vimeo.com/476389741.

\section{Online-Only Content}

Supplemental material is available online.

Supplementary Fig. 1. https://thejns.org/doi/suppl/10.3171/ 2020.9.FOCUS20672.

\section{Correspondence}

Akash J. Patel: Baylor College of Medicine, Houston, TX. akash. patel@bcm.edu. 